\title{
MEDICINA Y MORAL SEXUAL EN LA ESPAÑA DE PREGUERRA
}

\section{Raquel Alvarez Peláez}

En primer lugar, quisiera señalar algunos aspectos generales que creo deben ser tenidos en cuenta. Comenzando por el propio término de «sexualidad», concepto que puede indicar múltiples aspectos del ser sexual, y que puede referirse a formas o modalidades parciales de ese ser sexual. En general al referirnos a sexualidad nos referimos al comportamiento sexual. Pero más importante aún, y sobre todo para la medicina, es el concepto de "normalidad». Las variaciones de este concepto, vago pero manejado por todos como punto de referencia, han servido para el establecimiento de valoraciones dentro de la sociedad, para establecer los límites de la marginalidad y, en medicina, de lo patológico. En el caso del sexo es evidente, así como las variaciones que ha sufrido y sufre esta idea de «normalidad».

El establecimiento de un concepto más o menos estricto de normalidad obligará a una educación y control del comportamiento sexual rígido y extendido a muchos ámbitos: familiar, escolar, religioso, médico y social. Cuando el concepto de normalidad se hace más laxo y se tiende a no poner excesivos límites al comportamiento sexual, la educación y la patología se hacen menos importantes (1). El concepto de normalidad, la norma de comportamiento sexual existente en la España de comienzos de siglo, era tremendamente restrictivo, aceptando sólo la actividad sexual como actividad procreadora. Prácticamente no había referencias - más que en escritores malditos o en grupos marginalesal placer sexual. El llamado «instinto sexual» se identificaba con el llamado «instinto de procreación». Todo lo demás era pecado o, incluso, delito (2).

El sexo, la sexualidad, el comportamiento sexual en fin, tiene fuer- 
tes repercusiones sociales y económicas; por eso es una preocupación socio-política aunque se trate de un comportamiento de "puertas adentro». Del sexo depende la población, su cantidad y su calidad. El número, elemento esencial en todas las épocas, como vemos en la nuestra, y también la calidad, la necesidad de la regeneración de las razas o de la raza humana en general, como propugnaba la eugenesia.

El control de la procreación, pues, ha sido una necesidad esencial, y la forma de procrear se ha reglamentado desde la religión, desde la moral, desde la política y desde la medicina, impregnando religión y moral las opiniones científicas y técnicas de los otros sectores. Religión y moral puestas al servicio de ciertas ideologías pueden conseguir unas concepciones muy rígidas y reglamentadas del comportamiento sexual, como sucedía en España, cuya educación estaba, además, controlada también por la Iglesia.

Una sexualidad rígidamente orientada hacia la procreación dentro del matrimonio. Pecado de todo sentimiento fuera de esta norma. Castigo de todo comportamiento fuera de la regla, castigo legal de madres e hijos que concibieran o fueran concebidos fuera del matrimonio. Por esta razón todos los intelectuales y profesionales que más adelante lucharán por una educación sexual y una nueva moral sexual insistirán en la necesidad de evitar, en los jóvenes, el castigo de los comportamientos sexuales considerados inadecuados y los sentimientos de pecado y delito.

El hombre, tan pecador como la mujer, no tenía el mismo castigo legal y ni siquiera social, que ésta. La enorme estrechez de la norma hacía que la mayoría de los comportamientos estuviesen fuera de la norma. Y la diferencia en la consideración del pecador y delincuente según fuera hombre o mujer, establece la tan mentada y después combatida -en gran medida para eso quiere establecerse después la educación sexual- «doble moral sexual» (3). En el hombre se aceptaban el adulterio, el recurso a la prostitución y la paternidad fuera del matrimonio, aunque la madre y el niño fueran castigados legalmente, dejándoles sin derechos y culpándoles socialmente (4).

La norma, la normalidad sexual, se "enseñaba», se transmitía desde la infancia en la propia familia, en la educación, desde el púlpito o por medio del médico de la familia que aconsejaba o participaba muchas veces de los problemas sexuales de los niños o jóvenes. Existía, pues, una educación sexual, aunque no establecida como tal.

A lo largo de los primeros veinte años del siglo se comienzan a oír voces - y no sólo la de grupos políticos más radicales como los anar- 
quistas, o las de algunos individuos aislados y mal vistos, como los escritores de las llamadas «novelas eróticas», -Pedro Mata, Felipe Trigo, Hoyos y Vinent, Zamacois, etc.- de personas autorizadas y socialmente admitidas, fundamentalmente médicos, que luchan por una legislación más justa para madres e hijos ilegítimos, que buscan la protección de la maternidad y la infancia - Madrazo, González Alvarez, Martínez Vargas, etc.-. Por otra parte comienzan a circular ideas "positivistas", «biologicistas» y «naturalistas». La ciencia comienza a ser un patrón de valoración, y la sexualidad comienza a considerarse algo «natural».

En los años veinte los intentos de ruptura con la vieja y estrecha norma se hacen más evidentes. Son ya grupos de profesionales e intelectuales los que elevan su voz contra la vieja moral sexual o doble moral sexual. La vieja norma ya no sirve para los nuevos tiempos. En Europa, la guerra ha contribuido a cambiar, aunque fuese temporalmente, el papel de la mujer. Esta se siente con derechos y lucha por ellos. Se sube las faldas y se corta el pelo, conduce coches y juega al tenis. En la Sociedad Española de Higiene se discute, en definitiva, la redefinición de los conceptos de «femineidad" y «masculinidad».

La medicina está estrechamente ligada a las cuestiones de la sexualidad. Por una parte, por esa consideración de anormalidad que se daba y se da a todo comportamiento fuera de la normá, ligándolo, fundamentalmente, a alteraciones mentales. La psiquiatría, pues, es parte fundamental del juego del establecimiento de la normalidad sexual. Y son los psiquiatras quienes más se refieren no sólo a la patología sexual, sino a la educación sexual y a cómo debe realizarse, pues se está jugando con la salud mental. En 1930, la Liga Española de Higiene Mental propuso, como problema a discutir en el Congreso de 1932, el tema de la Educación Sexual, "por ser cuestión de gran importancia y que interesa a médicos, pedagogos, filósofos, juristas psiquiatras» (5). Por otra parte, la enorme difusión de las enfermedades venéreas hacía, indudablemente, que fuera importante la participación médica.

La gran cantidad de publicaciones que sobre el sexo y la sexualidad aparecen en España a partir, diría yo, del año 1926 - comienzo del final de la Dictadura de Primo de Rivera, quizás momento en que ya los intelectuales y profesionales decepcionados de ésta, en la que en cierta medida habían confiado, comienzan a reaccionar- permiten ver, por un lado, cuáles eran los temas claves que se debatían, y por otro lado, cómo se iban marcando las diferencias según las bases ideológicas y religiosas de cada uno. La radicalización y politización de los diversos sectores se hizo más evidente en los años treinta, como es lógico, cuan- 
do no era ya una lucha conjunta contra la Dictadura o contra la Monarquía.

En un principio, la lucha contra la doble moral sexual, contra la vieja moral sexual era unánime. Todos pensaban que debía ser transformada, y el mejor instrumento de transformación, además de la legislación adecuada, debía ser la educación sexual. La educación sexual debía ser el medio para que hombres y mujeres adquirieran un nuevo concepto, una nueva norma de sexualidad, que permitiera, a su vez, evitar los males de la época -enfermedades, abortos, infanticidios y abandonos, mala procreación, prostitución-y crear una nueva moral sexual más acorde con los tiempos. En este aspecto, la definición de papeles, la caracterización de femineidad y masculinidad eran muy importantes por el papel que la mujer comenzaba a jugar dentro de la sociedảd. Y es en estos aspectos donde más se notan las diferencias entre los médicos que se refieren a la educación sexual. Se manifiestản así las contradicciones de individuos que, llevados por unas concepciones políticas y una nueva concepción científica, han sido educados dentro de esa vieja moral sexual que atacan, y dentro de un catolicismo muy conservador y reaccionario. Esto les lleva a defender, por un lado, la necesidad de ciertos cambios que estén de acuerdo con la biología y con una sexualidad «natural», pero también a mantener ideas que afirman los papeles más tradicionales, los conceptos más retrógrados de "femineidad» $\mathrm{y}$ «masculinidad».

Ejemplo de esta actitud fue la de Marañón. Popular defensor de una nueva sexualidad, leído y escuchado por universitarios progresistas, su discurso en Amor, conveniencia y eugenesia, y en Tres ensayos sobre la vida sexual (6), se centra en demostrar, en una especie de sociobiología muy al uso en evolucionistas «al día», el maravilloso papel de la mujer como ser reproductor y como madre. Como tal, debe seleccionar al macho adecuado, y el macho adecuado en la sociedad es el bien situado social y económicamente. El hombre, a su vez, debe buscar a la hembra más adecuada para ser madre. Por tanto, con cualidades físicas y mentales apropiadas, sin necesidad de tener una posición social o económica superior. Aboga, pues, por el matrimonio de conveniencia - aunque la situación ideal es que también "sea por amorporque el fin último del sexo es la procreación. Por otra parte, desde el punto de vista de la educación sexual, la mujer debe ser educada para el matrimonio y la maternidad, pero no ya como antes. La cultura elemental, dice, debe ser igual en la niña y en el varón, y se debe realizar con coeducación. Pero, indica: «Lo menos es lo que aprende: esto siem- 
pre es lo de menos. Lo importante es asomarse al otro mundo, al de la vida del varón...» porque es una forma de adquirir naturalidad en las relaciones. Y agrega, «Lo fundamental es que ninguna de ellas será ya la madre pasiva, resignada y fanática, víctima de su propio instinto generoso". Y como está demostrado que, aunque hombre y mujer deben ser considerados iguales porque todos los trabajos son válidos, la realidad es que son diferentes biológicamente, no es necesario para la tarea fundamental de la hembra que ésta realice estudios universitarios. Senala entonces que, aunque el hombre ha limitado arbitrariamente la capacidad de acción de la mujer, al obrar así «no creaba en realidad una injusticia, sino tan sólo intrepretaba abusivamente un estado de desigualdad natural e inmodificable entre ambos sexos». "Como regla general - dice- no creemos admisible el que la cultura definitiva de la mujer sea la misma del varón. $\mathrm{Ni}$, por tanto, que su actividad social se encauce por los mismos carriles que la del hombre» (7).

Esta postura tradicional con respecto a la mujer era mantenida por gran número de médicos. La figura de maternidad, defendida más modernamente en cuanto a sus derechos, incluso fuera del matrimonio, seguía siendo un elemento esencial, porque el problema de la cantidad y calidad de la población continuaba siendo crucial.

Las transformaciones fundamentales de los conceptos de sexualidad, y dentro de ella, de masculinidad y femineidad, necesitan de transformaciones económicas y sociales para poder producirse realmente. Para los grupos de pensamiento más a la izquierda, y que buscaban una transformación del orden social, la mujer debía adquirir el derecho a su libertad económica y a su propia sexualidad. Era lo que manifestaban los anarquistas, aunque defendiendo o protegiendo la cuestión de la procreación. Para muchos de los republicanos, liberales y más o menos izquierdistas que luchaban por el proyecto republicano, la educación, y dentro de ella la educación sexual, era el medio de transformación, o más bien reforma del orden social. Un grupo de médicos, muchos de ellos psiquiatras, lleva el problema a la calle, a la discusión pública y en su día a las Cortes Constituyentes - es interesante recordar que el 10 por 100 de los diputados de estas cortes eran médicosentre ellos Juarros y su lucha abolicionista, Lafora y sus polémicas y sus escritos sobre educación sexual, y algunos otros que participan en el Primer Curso Eugénico Español —suspendido por la dictadura a instancias de la iglesia, en 1928-y después, en 1933, en las Jornadas Eugénicas (8).

Esta lucha, y las manifestaciones de estos médicos, eran expresión 
de un deseo de reformar un orden social, de romper con una moral estrecha y caduca. Sus manifestaciones sobre la sexualidad, y lo que pretenden con la educación sexual, además de integrar los nuevos conocimientos científicos y médicos, manifiesta sus distintas orientaciones en cuanto a qué cambios desean y hasta dónde quieren llegar con esa reforma social.

Veamos ahora algunos de los contenidos de esa nueva moral sexual que se quiere.

En general hay unanimidad en la necesidad de que exista una educación sexual en cierta medida organizada, y conducida por especialistas. Indudablemente los padres y la familia son elementos esenciales de esa educación -así Hildegart en su folleto sobre educación sexual comienza con la educación de los padres (9)- pero debe existir una orientación profesional por parte de pedagogos y sobre todo de médicos. Y son los psiquiatras, no los que imparten esa educación desde la infancia, pero sí quienes opinan y dan nuevas normas sobre el comportamiento sexual. Son ellos los que deben decir qué es «normal»y que puede permitirse, y qué es «anormal» y debe evitarse, no ya con consideraciones religiosas, sino con afirmaciones científicas.

En general se considera que la educación sexual debe impartirse al niño y al joven. Aquí se plantea un primer conflicto, el de saber cuándo comienza la sexualidad del ser humano. Para los escasos adeptos, o al menos conocedores, de Freud la sexualidad existe como impulso vital desde que se origina el ser. Para la generalidad la sexualidad comienza en la pubertad, entre los 14 y los 21 años. Si es anterior, cae ya en la «anormalidad», se le llama «madurez sexual prematura» o precocidad sexual, como indica Lafora en su Pedagogía Sexual (10). Indica también Lafora que para algunos pedagogos es parte de la «dismoralidad constitucional» o «locural moral». Se dice que los niños con esta anormalidad son también mentirosos, ladrones, hiperfantásticos. Lafora indica que la asociación no es constante, pero sí frecuente. Se asocian las «anormalidades» sexuales con la delincuencia. Aunque también reconoce Lafora la existencia de factores ambientales que pueden llevar a la precocidad en el comportamiento, sin que exista una verdadera precocidad sexual: libertad excesiva de los niños y necesidad económica en los medios proletarios (11).

Para Angel Suils, seguidor de Freud, el instinto sexual existe desde el primer instante de la vida y comienza a desarrollarse en la relación con la madre y con el padre. No voy a desarrollar aquí el discurso freudiano - mejor asimilado en este caso que en el de Juarros- sino que 
lo que señala Suils son los múltiples caminos que a partir de aquí pueden surgir, y las múltiples trampas que aparecen en el desarrollo de una sexualidad «normal». Y dice: «De esta manera - por las múltiples trampas de mala información por compañeros o de las malas influencias de profesores- caen en la enfermedad muchísimos individuos» (12). Por tanto, la educación sexual es necesaria para evitar anormalidades. Para él falta educación sexual a todos los niveles, y sobran malas influencias familiares y ambientales. «De todas estas maneras, dice Suils, el inconsciente resulta lesionado, originando las distintas psiconeurosis» (13).

En resumen, para Suils la verdadera educación sexual sería la siguiente:

«La verdadera conducción está en la activa adaptación paterna de los instintos de sus hijos a las necesidades de la civilización, en el terreno moral, sin enseñarles desde el principio la conducta de los veinte años: en el religioso, no empezar por dogmas que en la misma edad adulta habrán de serles indescifrables; en el terreno sexual, menos ignorancia cultivada y menos cultivo de la hipocresía» (14).

Su último apartado se dedica a buscar una nueva moral sexual "postpsicoanalítica», que cifra en que el deseo moral es una fuerza instintiva tan fuerte como el instinto sexual. Hay que buscar la coherencia entre ambos, buscando la «naturalidad».

Los psiquiatras de ideas más conservadoras parecen centrar su interés en una educación sexual, por un lado del hombre con respecto a las enfermedades de transmisión sexual -así se discutía, dentro de la búsqueda de medidas eugenésicas, el establecimiento o no de un certificado prenupcial obligatorio para el hombre (15)-, y de la mujer, enseñándole a tener una buena preparación para el matrimonio y la maternidad.

Los médicos, digamos que filo-anarquistas, mantenían posturas más radicales con respecto a la libertad de la mujer y a su derecho a la sexualidad, así como al trabajo. Las propuestas, entonces, iban por el camino de establecer leyes y medidas que permitieran la protección de la mujer embarazada, la protección de su puesto de trabajo y el consejo médico. La revista Estudios publicaba gráficos enseñando los ciclos menstruales de la mujer y los períodos de fertilidad, así como la anatomía de los aparatos reproductores, poniendo como material gráfico fotografías de personas desnudas en el campo o la playa, todo ello con una orientación naturista (16).

En definitiva, e intentando resumir la postura de algunos de los psi- 
quiatras que hemos elegido como representativos de varias de las actitudes hacia la sexualidad que en la España de los años veinte y treinta se mantenían, podríamos decir lo siguiente: Lafora quería, o así lo parece por sus escritos, una nueva moral sexual que permitiera, si no el matrimonio a prueba, algo parecido. Que se pudieran formar matrimonios bien avenidos en el sexo y en el amor, que tuvieran una procreación sana de cuerpo y espíritu. Consideraba que para conseguir este fin se debía integrar la educación sexual a todos los niveles y dentro de las asignaturas normales de la enseñanza, no como cursos específicos. Por otra parte, consideraba necesaria la sublimación del impulso sexual en fines sociales, para evitar patologías y relaciones sexuales sin amor. Suils quería evitar las múltiples patologías sexuales que la falta de educación y conocimiento permitía que se desarrollaran. Juarros quería, por encima de todo, la abolición de la prostitución y la consecución del amor placentero y a la vez puro. Fernández Sanz quería mantener a ultranza los papeles tradicionales de femineidad y masculinidad, y, «la eterna e inevitable guerra de los sexos». Marañón, por su parte, deseaba que se mantuvieran los papeles tradicionales del hombre y la mujer, pero con madres algo menos torpes y aburridas. El ginecólogo Vital Aza - muchos ginecólogos participaban en estos debates- decía «no queremos hembras gozadoras estériles», manteniendo la identidad de sexo y procreación. Martí Ibáñez, Jesús Puente, y algunos otros anarquistas o cercanos a ellos, el derecho de la mujer a la sexualidad, aunque la necesidad inevitable de proteger la procreación les llevará a apoyar el matrimonio civil.

Por fin, la guerra civil y el franquismo ahogaron todas estas alentadoras polémicas y todas las posturas moderadamente progresistas, para dejar al país sumido en la más oscurantista moral sexual.

\section{NOTAS}

(1) Es interesante consultar, para conocer los conceptos de discusiones actuales sobre el sexo y la sexualidad, la obra editada por SHELP, Earl E. (1987): Sexuality and Medicine (2 vol), Dordrecht, Holanda. Algunos de los capítulos son especialmente interesantes para nuestra aproximación a la cuestión de la norma en la sexualidad, como el Prefacio de SABLE, Alan (1987): Changing Conceptions of Human Sexuality, pp XI-XXIV, vol. I.

(2) A principios de siglo sólo había algunas publicaciones, en general dentro de la órbita anarquista, que se insertaban dentro de las corrientes de liberación sexual. Entre ellas Salud y Fuerza, revista mensual ilustrada de la Liga de Regeneración Humana, Liga creada a partir de la del mismo nombre liderada en Francia por Paul Robin. La revista llevaba por lema «Procreación consciente y.limitada», defendiendo pues el neomalt- 
husianismo y difundiendo los métodos anticonceptivos del momento: Los «Conos preservativos del embarazo y de las enfermedades sexuales, del Dr. Mascaux»; el "Formolodor Veignault. Esterilizante el más poderoso, propio para las inyecciones diarias. Medalla de oro en la Exposición de Higiene de París», o «El Obturador Uterino. Evita los embarazos - contraindicados-, Depositario: Dr. Queral», etc. Luis Bulffi, el fundador de la revista -fue fundada en 1904 y apareció en 1907- dirigía además una editorial que publicaba folletos y pequeños libros sobre los mismos temas, entre ellos uno del que era autor él mismo, Huelga de Vientres, llamando, pues, también al control de natalidad.

(3) En estos aspectos de la sexualidad del hombre y la mujer pesaban dos tipos de consideraciones importantes: por un lado, el establecer una "maternidad consciente» que permitiera una buena procreación y una disminución de la enorme mortalidad infantil -además de los abortos e infanticidios-que en España existía; y un mayor control de las enfermedades venéreas buscando una disminución de la prostitución sin necesidad de establecer un reglamento de ella que resultaba inútil y contra el que también lucharon muchos de los médicos eugenistas, fundamentalmente, como gran adalid, el Dr. César Juarros.

(4) Luis Jiménez de Asúa y Joaquín Noguera fueron grandes luchadores que defendieron el derecho de la mujer a tener los hijos en condiciones dentro y fuera del matrimonio, así como las leyes de aborto y divorcio. El Curso Eugénico de 1928 se suspendió justamente como consecuencia de la conferencia de Jiménez de Asúa sobre estos temas, "Aspecto Jurídico de la Maternidad Consciente», que indignó a las fuerzas católicas, como se manifestó a través de los artículos del periódico El Debate. Decía Jiménez de Asúa en esta conferencia: "Este Curso de Eugénica responde a una realidad ambiente. Nos hallamos en momentos en que España barre con prisas superlativas toda la fronda donde se ocultan los rancios prejuicios que afectan al problema sexual. Hace un decenio, acaso habría sido prematuro organizar un curso como éste; cuestiones que entonces no podían ser esclarecidas en público, hoy se debaten a presencia de las gentes con decoro en el lenguaje pero con valentía de conceptos que nuestros padres no sospecharon" $\mathrm{J}_{\mathrm{I}}$ MÉNEZ DE ASUA, L. (1934): «Aspecto jurídico de la maternidad consciente», en Genética, Eugenesia y Pedagogía Sexual, Libro de las primeras Jornadas Eugénicas Españolas, $\mathrm{Ma}$ drid, pp. 333-341. Si se quiere más información sobre el papel de la eugenesia en España se puede consultar de AlvarEZ, R. (1988): «Origen y desarrollo de la eugenesia en España", en Ciencia y Sociedad en España, Madrid, pp. 179-204.

(5) Así lo indica el psiquiatra Prados Such en su artículo de El Sol «Hacia una nueva moral sexual».

(6) Marañon, G. (1926): Tres ensayos sobre la vida sexual. Sexo, trabajo y deporte. Maternidad y feminismo. Educación sexual y diferenciación sexual. Madrid. Y MARAÑon, G. (1927): Amor, conveniencia y eugenesia, Madrid. Ambos libros tuvieron gran éxito y se hicieron de ellos numerosas ediciones.

(7) Marañon, G. (1920): «Biología y Feminismo», en El Siglo Médico, 10 de abril.

(8) Ya hemos hablado del Primer Curso Eugénico Español y su suspensión instigada por la jerarquía eclesiástica. Las Primeras Jornadas Eugénicas Españolas se realizaron durante 1933 con la participación de numerosos médicos, juristas, pedagogos y escritores y están publicadas en dos volúmenes: NORIEGA, E. y LuIS HuERTA (1934): Genética, Eugenesia y Pedagogía Sexual, Madrid.

(9) En los años treinta se funda en España la Liga para la Reforma de la Moral Sexual sobre Bases Cientificas, filial de la misma organización internacional en la que figuraban personajes como Magnus Hirschfeld y Havelock Ellis. En España comenzó tenien- 
do a Marañón como presidente y a la joven y famosa Hidegart como secretaría. Esta Liga publicaba la revista Sexus, en la que se contenían artículos de los más destacados médicos españoles y extranjeros que se preocupaban por el tema. La propia Hidelgart publicó algunos libros y numerosos folletos, entre los que se encuentra el titulado Educación Sexual, que comienza justamente refiriéndose en primer lugar a la necesidad de educar a los padres para conseguir una buena educación sexual de la juventud.

(10) Lafora, G. Rodriguez (1934): «Pedagogía Sexual» en Genética, Eugenesia y Pedagogía Sexual, Madrid, vol.I, pp. 107-123. También en LAFORA, G. R. y ComAS, Margarita (1947): La Educación Sexual y la Coeducación de los Sexos, Buenos Aires.

(11) LAFORA (1934): p. 107.

(12) SuILS, Angel (1934): «Consideraciones eugénicas prácticas según la psicopatología del subconsciente», "I-Higiene mental en los progenitores. II-Higiene mental en el niño. III-Hacia una moral postpsicoanalítica». En Genética, Eugenesia y Pedagogía sexual, Madrid, vol. II, pp. 91-116.

(13) Ibidem, p. 106

(14) Ibidem, p. 108

(15) La cuestión del establecimiento de un certificado médico prenupcial tiene una larga historia en España - y en casi todos los países europeos y americanos-pues desde comienzos de siglo se sintió la necesidad de utilizar un mecanismo de este tipo para el control de esas enfermedades que eran una verdadera plaga; la sífilis y la tuberculosis. A ello se agregó la actitud eugénica de controlar todas las taras que los futuros progenitores pudieran llevar al matrimonio y por ende a la progenie. En definitiva, podemos decir que en pocos casos este tipo de certificación llegó a ser obligatoria, pero, sin embargo, la utilidad de realizar un examen médico previo al matrimonio prendió en muchos países y se integró a la medicina preventiva. Esto es muy notorio en algunos países de América del Sur. En España las propuestas de su establecimiento obligatorio comenzaron en 1915, y en los años posteriores hubo grandes discusiones sobre el tema, sin que se llegara a legislar. Era parte de los temas prácticos de la eugenesia.

(16) En Estudios se publicaba el «Consultorio psíquico-sexual» conducido por Félix Martí Ibáñez, el conocido psiquiatra, eugenista y anarquista que en 1937 llegó a la Dirección de Sanidad de la Generalitat de Cataluña. 